\title{
Turbulent-diffusion Mediated CO Depletion in Weakly Turbulent Protoplanetary Disks
}

\section{Citation}

Xu, Rui, Xue-Ning Bai, and Karin Öberg. 2017. "Turbulent-Diffusion Mediated CO Depletion in Weakly Turbulent Protoplanetary Disks." The Astrophysical Journal 835 (2) (January 27): 162. doi:10.3847/1538-4357/835/2/162.

\section{Published Version}

doi:10.3847/1538-4357/835/2/162

\section{Permanent link}

http://nrs.harvard.edu/urn-3:HUL.InstRepos:32192695

\section{Terms of Use}

This article was downloaded from Harvard University's DASH repository, and is made available under the terms and conditions applicable to Other Posted Material, as set forth at http:// nrs.harvard.edu/urn-3:HUL.InstRepos:dash.current.terms-of-use\#LAA

\section{Share Your Story}

The Harvard community has made this article openly available.

Please share how this access benefits you. Submit a story.

\section{Accessibility}




\title{
Turbulent-diffusion Mediated CO Depletion in Weakly Turbulent Protoplanetary Disks
}

\author{
Rui Xu (徐睿) ${ }^{1}$, Xue-Ning Bai $^{2}$, and Karin Öberg ${ }^{3}$ \\ ${ }^{1}$ Department of Astrophysical Sciences, Princeton University, Princeton, NJ 08544, USA; ruix@ princeton.edu \\ ${ }^{2}$ Institute for Theory and Computation, Harvard-Smithsonian Center for Astrophysics, 60 Garden Street, MS-51, Cambridge, MA 02138, USA; xbai@cfa.harvard.edu \\ ${ }^{3}$ Harvard-Smithsonian Center for Astrophysics, 60 Garden St., MS-16, Cambridge, MA 02138, USA; koberg@ cfa.harvard.edu \\ Received 2016 September 2; revised 2016 December 20; accepted 2016 December 22; published 2017 January 27
}

\begin{abstract}
Volatiles, especially $\mathrm{CO}$, are important gas tracers of protoplanetary disks (PPDs). Freeze-out and sublimation processes determine their division between gas and solid phases, which affects both which disk regions can be traced by which volatiles, and the formation and composition of planets. Recently, multiple lines of evidence have suggested that CO is substantially depleted from the gas in the outer regions of PPDs, i.e., more depleted than would be expected from a simple balance between freeze-out and sublimation. In this paper, we show that the gas dynamics in the outer PPDs facilitates volatile depletion through turbulent diffusion. Using a simple 1D model that incorporates dust settling, turbulent diffusion of dust and volatiles, as well as volatile freeze-out/sublimation processes, we find that as long as turbulence in the cold midplane is sufficiently weak to allow a majority of the small grains to settle, $\mathrm{CO}$ in the warm surface layer can diffuse into the midplane region and deplete by freeze-out. The level of depletion sensitively depends on the level of disk turbulence. Based on recent disk simulations that suggest a layered turbulence profile with very weak midplane turbulence and strong turbulence at the disk surface, $\mathrm{CO}$ and other volatiles can be efficiently depleted by up to an order of magnitude over Myr timescales.
\end{abstract}

Key words: protoplanetary disks - turbulence

\section{Introduction}

Protoplanetary disks (PPDs) consist of gas and dust. Both components play a major role in planet formation through dynamical processes in the gaseous disk, as well as physical and chemical coupling between gas and dust components. The dust can be probed via the disk spectral energy distribution and resolved dust continuum emission up to millimeter/centimeter grain sizes (Andrews 2015). Despite uncertainties in dust opacity, dust mass can be derived from submillimeter continuum flux (e.g., Williams \& Cieza 2011). There is no corresponding direct constraint on the gas because molecular hydrogen hardly radiates, the gas mass is instead usually estimated by assuming a canonical gas-to-dust mass ratio of 100 from the interstellar medium (e.g., Bohlin et al. 1978), leading to large uncertainties.

Recently, a number of works have attempted to measure the gas content of PPDs using $\mathrm{CO}$ and its isotopologues (e.g., Bruderer et al. 2012; Williams \& Best 2014; Ansdell et al. 2016; Eisner et al. 2016; Kama et al. 2016a, 2016b). As a volatile species, $\mathrm{CO}$ freezes out onto dust grains in the cold midplane regions of the outer PPDs, while it remains in the gas phase in the warmer disk surface layer (e.g., Henning \& Semenov 2013). These studies, which incorporate CO freezeout and different levels of disk chemistry, found that if one assumes a standard gas to dust ratio and a canonical $\mathrm{CO} / \mathrm{H}_{2}$ ratio of $\sim 10^{-4}$ (e.g., Frerking et al. 1982; Ripple et al. 2013), $\mathrm{CO}$ is frequently underabundant by a factor of $\gtrsim 10$ in the warm disk surface layer. This result holds also if isotopologueselective photodissociation is taken into account (Miotello et al. 2014; Schwarz et al. 2016). Therefore, either CO is intrinsically depleted, or the gas-to-dust mass ratio is significantly lower than the standard value.

Theoretically, both scenarios are plausible. The gas-to-dust ratio can be reduced via disk wind, where mass loss from the disk surface primarily removes gas instead of dust (Gorti et al. 2015; Bai 2016). In the mean time, through chemical processes, a significant fraction of carbon can be converted to complex organic molecules over the disk lifetime (Bergin et al. 2014, 2016; Yu et al. 2016). The presence of CO depletion is supported at least in the case of TW Hya (Favre et al. 2013; Du et al. 2015), where a constraint on the disk gas mass is available from HD observations (Bergin et al. 2013).

Volatile depletion has also been inferred in the case of water, whose freeze-out temperature is much higher. Based on Spitzer mid-infrared observations of $\mathrm{H}_{2} \mathrm{O}$ lines (Carr \& Najita 2008; Salyk et al. 2008), Meijerink et al. (2009) showed that water vapor abundance at the disk surface is sharply truncated beyond $\sim 1$ au, inconsistent with pure chemical models (e.g., Glassgold et al. 2009). They hypothesized that beyond $\sim 1$ au, warm water vapor at the disk surface diffuses vertically toward the midplane by turbulence and freezes-out onto the solids to account for the truncation. This water vapor depletion mechanism by turbulent diffusion is related to the "cold-finger effect" of Stevenson \& Lunine (1988), but working in the vertical direction. Using Monte Carlo simulations of dust/ vapor dynamics, Ros \& Johansen (2013) and Krijt et al. (2016) indeed found rapid depletion of water vapor in the surface layer of inner PPDs near the water ice line. They further showed that the depletion process strongly promotes grain growth, and hence planetesimal formation.

In this paper, we apply the picture of turbulent-diffusion mediated volatile depletion to $\mathrm{CO}$. Note that this picture has also been suggested by Kama et al. (2016b) recently to interpret carbon depletion based on ALMA observations of CO and [C I] lines from two PPDs. We focus on the outer regions of PPDs $(\gtrsim 10-30 \mathrm{au})$, which are where most of the CO mass resides, and are also where significant $\mathrm{CO}$ depletion has been observationally measured. Compared to the inner disk, the outer disk is characterized by much lower gas density and much longer dynamical timescales. Correspondingly, dust grains of the same size are more loosely coupled to the gas in the outer disk, and settle more strongly toward the midplane, 


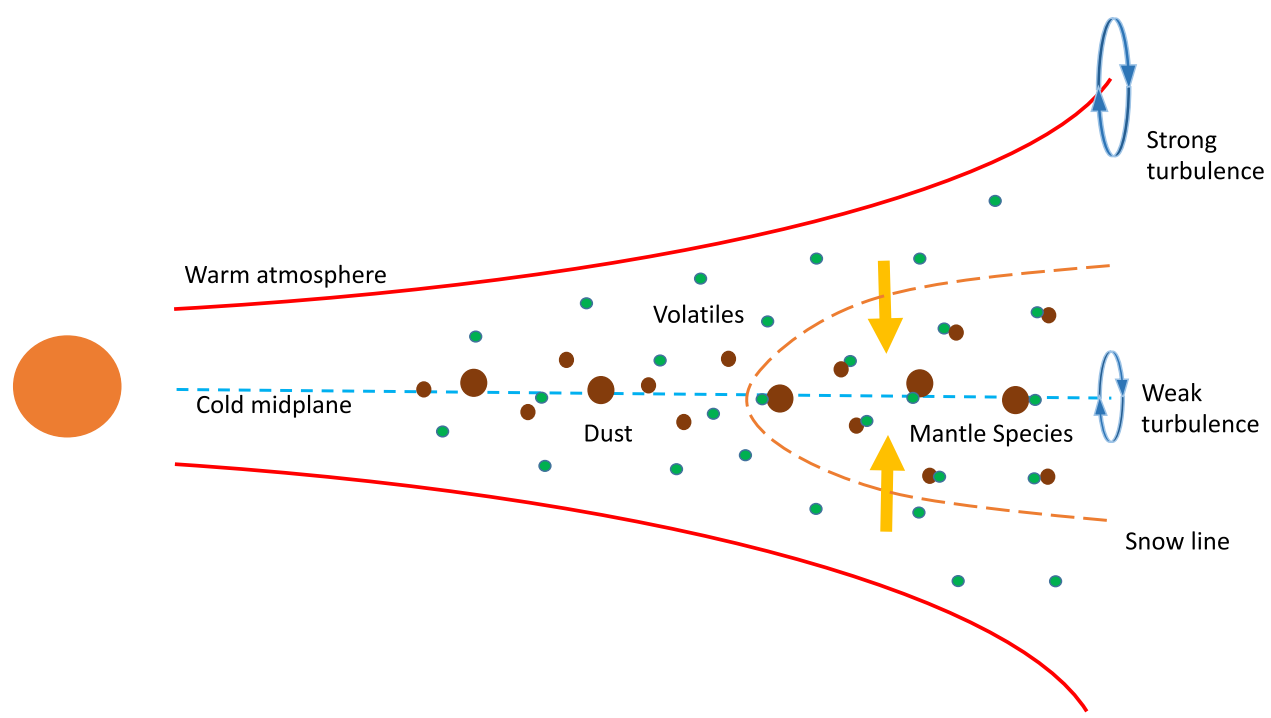

Figure 1. Schematic picture of the turbulent diffusion mediated volatiles (CO) depletion in PPDs. Freeze-out of CO on the dust grain surface at the low-temperature midplane allows surface $\mathrm{CO}$ to turbulently diffuse down to the midplane, which further freezes out onto the grains. Dust grains settle to the midplane without mixing back to the disk surface due to weak midplane turbulence, leading to systematic $\mathrm{CO}$ depletion.

which, as this paper shows, has a large impact on volatile depletion.

In this work, we present a simple semi-analytical model for the evolution of $\mathrm{CO}$ abundances to quantify the efficiency of gas-phase $\mathrm{CO}$ depletion. It incorporates dust settling, turbulent diffusion, adsorption (freeze-out), and thermal desorption (sublimation) processes. We do not attempt to model the entire disk in full scale, but restrict ourselves to a simple onedimensional (1D) model in the vertical dimension. Our goal is to demonstrate and clarify the relevant physics, which can be incorporated into more sophisticated models in the future. We highlight that the level of disk turbulence we adopt is motivated from recent gas dynamic simulations of the outer PPDs (Simon et al. 2013; Bai 2015) that take into account more realistic disk physics: the level of turbulence in the outer disk is layered, with strong turbulence in the warm disk surface layer and much weaker turbulence in the midplane region (see Section 2 for more details). We will show that this layered structure of turbulence facilitates the depletion of gas-phase CO.

We describe the basic picture of turbulent-diffusion mediated $\mathrm{CO}$ depletion, as well as our physical model in Section 2. Model results are presented in Section 3. In Section 4, we summarize and discuss the caveats and applications.

\section{Model Description}

We are mainly concerned with the outer regions of PPDs, which are characterized by low temperatures and weak turbulence in the midplane, and higher temperatures and levels of turbulence in the disk surface layers. In Figure 1, we illustrate the basic picture of turbulent-diffusion mediated $\mathrm{CO}$ depletion, and elaborate below.

Due to external heating by stellar UV and X-rays, PPDs exhibit a vertical temperature gradient. Disk temperature is sufficiently low in the midplane $(\lesssim 20 \mathrm{~K}$, e.g., Bisschop et al. 2006; Qi et al. 2013) for CO to freeze out onto dust grains, while the disk surface is much warmer so that $\mathrm{CO}$ remains largely in gas phase (e.g., Glassgold et al. 2004; Kamp \& Dullemond 2004; Walsh et al. 2010; Rosenfeld et al. 2013). This dividing line for freeze-out is sometimes referred to as the "atmospheric snow line," which is approximately two horizontal surfaces above/below the midplane. The radial snow line, which is more commonly known, corresponds to the location in the midplane region where temperature transitions through the $\mathrm{CO}$ freeze-out temperature. It smoothly joins the atmospheric snow line toward larger radii, as shown in Figure 1. We consider the regions beyond the radial $\mathrm{CO}$ snow line, where only atmospheric snow lines are present.

Another key ingredient in the picture is the level of turbulence in PPDs. Due to the weakly ionized nature of PPD gas, its dynamics suffers from strong non-ideal magnetohydrodyamic effects (see Turner et al. 2014, p. 411 for a review), which can significantly weaken or suppress turbulence generated from the magnetorotational instability (MRI, Balbus \& Hawley 1991). The gas dynamics in the outer PPD is relatively simple because ambipolar diffusion (AD) is the only dominant non-ideal MHD effect. It has been shown that the MRI in the disk midplane is substantially damped by $\mathrm{AD}$, leading to weak turbulence (Bai \& Stone 2011). On the other hand, the disk surface layer is likely much better ionized due to far-UV radiation (PerezBecker \& Chiang 2011), leading to strong MRI turbulence (Simon et al. 2013; Bai 2015).

The basic mechanism of turbulent-diffusion mediated $\mathrm{CO}$ depletion is easily explained: freeze-out of $\mathrm{CO}$ in the cold midplane creates a sink in gas-phase $\mathrm{CO}$, leading to a diffusive flux of $\mathrm{CO}$ gas into the midplane due to turbulent mixing, which then quickly freezes out.

One caveat to the above picture is that the role of turbulence is two-fold. Besides driving a diffusive flux of gas-phase $\mathrm{CO}$ down to the midplane, turbulence can also potentially bring the dust to the warmer disk surface. This would allow mantlephase $\mathrm{CO}$ to sublimate and be released back to the gas phase. If this process is efficient, it may largely cancel the aforementioned CO depletion process. In a scenario where the disk midplane region is only very weakly turbulent, it is possible that the vast majority of the dust grains are strongly settled toward the midplane and never rise to the surface, then the gasphase $\mathrm{CO}$ would be systematically depleted. 

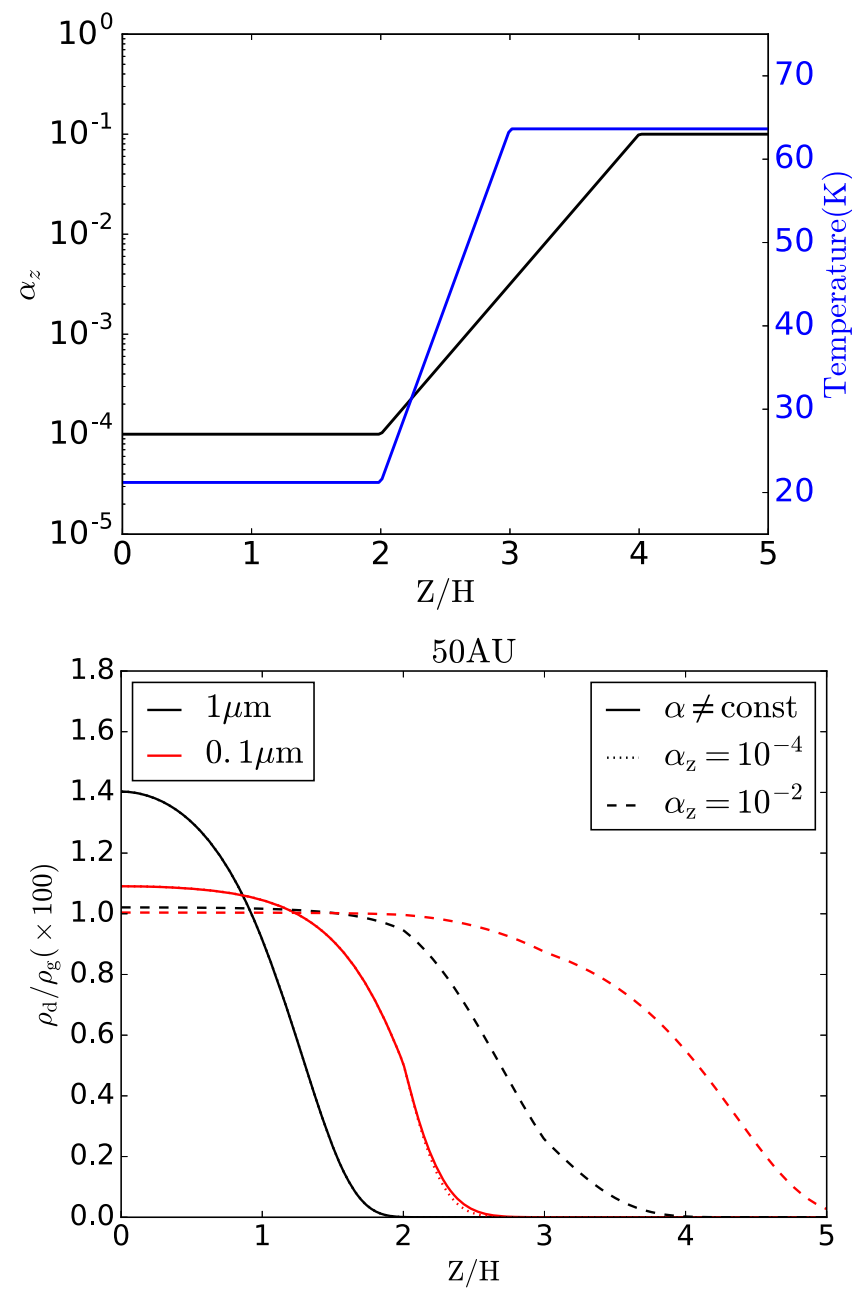

Figure 2. Top: vertical profile of the turbulent diffusion coefficient $\alpha_{z}$ adopted in this work and vertical temperature profile at $50 \mathrm{au}$. Bottom: vertical profiles of single-sized $1 \mu \mathrm{m}$ (black) and $0.1 \mu \mathrm{m}$ (red) dust at 50 au with layered turbulent diffusivity profile (solid lines, $\alpha_{z}$ from the top panel), constant $\alpha_{z}=10^{-4}$ (dotted lines) and constant $\alpha_{z}=10^{-2}$ (dashed lines). Dust to gas mass ratio is $10^{-2}$.

\subsection{Disk Model and Dust Properties}

Motivated by observations, we consider a thin disk model with surface density $\Sigma(r)=500 r_{\mathrm{au}}^{-1} \mathrm{~g} \mathrm{~cm}^{-2}$ and a midplane temperature profile $T_{\text {mid }}(r)=150 r_{\mathrm{au}}^{-1 / 2} \mathrm{~K}$ where $r_{\mathrm{au}}$ is disk radius measured in au (Andrews \& Williams 2007; Andrews et al. 2009). We focus on the outer regions of PPDs at radii $R \gtrsim 30 \mathrm{au}$ and choose a fiducial radius of $R=50 \mathrm{au}$, which is well outside of the radial CO snowline. Disk vertical temperature profiles are poorly constrained observationally (e.g., Rosenfeld et al. 2013), and for simplicity we assume that the temperature is $T=T_{\text {mid }}(r)$ within two disk scale heights $\left(2 H\right.$, with $H$ measured at disk midplane) ${ }^{4}$ It increases linearly to $3 T_{\text {mid }}(r)$ in one scale height and remains at $3 T_{\text {mid }}(r)$ beyond $z= \pm 3 H$ (see Figure 2(a)). Vertical density structure is then simply determined from hydrostatic equilibrium. To mimic the results of the layered turbulence profile, we employ a toy $\alpha_{z}$ profile where it increases exponentially from $10^{-4}$ starting at $2 H$ to $10^{-1}$ at $4 H$ (see Figure 2(a)), which

\footnotetext{
4 Disk scale height $H$ is defined as $H=c_{s} / \Omega_{K}$, where $\Omega_{K}$ is the midplane Keplerian frequency, $c_{s}=\sqrt{k_{B} T_{\text {mid }}(r) / \mu m_{p}}$, is the midplane sound speed, with mean molecular weight taken to be $\mu=2.34$.
}

is motivated from the vertical turbulent velocity profiles from the outer PPD simulations of Bai (2015).

Dust grains interact with the gas via aerodynamic drag, with drag force given by $F_{\text {drag }}=m_{\mathrm{d}}|\Delta \boldsymbol{v}| / t_{\text {stop }}$, where $m_{\mathrm{d}}$ is dust mass, $\Delta v$ is the relative velocity between dust and gas. In the outer disk, gas drag is in Epstein's regime (Epstein 1924), with stopping time $t_{\text {stop }}$ given by $t_{\text {stop }}=\rho_{s} a / \rho_{\mathrm{g}} c_{s}$, where $\rho_{\mathrm{g}}$ is gas density, $\rho_{s}=2 \mathrm{~g} \mathrm{~cm}^{-2}$ is the dust solid density, $a$ is dust grain size. It is more convenient to use the dimensionless stopping time $\tau_{s}=t_{s} \Omega_{K}=\rho_{s} \Omega_{K} a / \rho_{\mathrm{g}} c_{s}$.

In the vertical dimension, dust mass density $\rho_{d}$ satisfies the continuity equation (Takeuchi \& Lin 2002)

$$
\frac{\partial \rho_{\mathrm{d}}}{\partial t}+\nabla \cdot\left(\rho_{\mathrm{d}} v_{\mathrm{d}}+j_{\mathrm{d}}\right)=0
$$

where $v_{d}$ is the dust settling velocity, $j_{d}$ is dust diffusive flux given by

$$
j_{\mathrm{d}}=-\frac{\rho_{\mathrm{g}} D_{z}}{S_{\mathrm{c}}} \frac{\partial}{\partial z}\left(\frac{\rho_{\mathrm{d}}}{\rho_{\mathrm{g}}}\right) .
$$

In the above, $D_{z}$ is the diffusion coefficient, which we parameterize as $D_{z}=\alpha_{z} c_{s} H$ (Shakura \& Sunyaev 1973). The Schmidt number $S_{\mathrm{c}}$ represents the strength of coupling between dust and the gas, which is approximated as $1+\tau_{s}^{2}$ for turbulent eddy time $\sim \Omega_{K}^{-1}$ (Youdin \& Lithwick 2007). The settling velocity is given by

$$
v_{\mathrm{d}}=-\Omega_{K} z \tau_{s} / S_{\mathrm{c}}
$$

The extra factor $S_{\mathrm{c}}$ guarantees that in the limit $\tau_{s} \ll 1, v_{\mathrm{d}}$ is simply the dust terminal velocity. In the opposite limit $\tau_{s}>1$, dust particles undergo damped oscillation about the midplane with mean settling velocity $\sim 1 / \tau_{s}$ that is also captured by the expression.

In steady state, the solution to Equation (1) can be expressed as

$$
\left(\frac{\rho_{\mathrm{d}}}{\rho_{\mathrm{g}}}\right)_{z=z_{0}}=\left(\frac{\rho_{\mathrm{d}}}{\rho_{\mathrm{g}}}\right)_{z=0} \exp \left[\int_{0}^{z_{0}}-\frac{\tau_{s}\left(z^{\prime}\right) z^{\prime}}{\alpha_{z}\left(z^{\prime}\right) H^{2}} d z^{\prime}\right] .
$$

Note that both $\tau_{s}$ and $\alpha_{z}$ depend on $z$, and substantial dust settling is realized around the height where $\tau_{s}(z) \sim \alpha_{z}(z)$. For constant $\tau_{s}$ and $\alpha_{z}$, the dust distribution becomes a Gaussian with scale height is $\sqrt{\alpha_{z} / \tau_{s}} H$, recovering the result of Youdin \& Lithwick (2007) in the limit of $\alpha_{z}<\tau_{s}$.

In Figure 2(b), we show the dust density profile at $50 \mathrm{au}$, and compare with constant $\alpha_{z}$ profiles. For constant $\alpha_{z}=10^{-4}$, even sub-micron sized dust settles to within $\pm 2 H$. For stronger turbulence $\alpha_{z}=10^{-2}$, however, dust particles are stirred to well above $\pm 3 H$ from the midplane. Using our adopted layered $\alpha_{z}$ profile, the results are almost identical to the constant $\alpha_{z}=10^{-4}$ case, because the layered profile shares the same $\alpha_{z}$ in the bulk of the dust layer.

The above calculation assumes a single dust size, but in reality, dust grains collide with each other, resulting in a size distribution from coagulation and fragmentation (Birnstiel et al. 2011). In this work, We first consider a single grain size to clarify the most important physics. We then proceed with a more realistic size distribution in the form of $n_{d}(a) \propto a^{\beta}$ where $n_{d}(a) d a$ is the grain number density between size $a$ and $a+d a$. We take $\beta=-3.5$ as in the standard MRN size 
distribution (Mathis et al. 1977). The largest grain size is fixed to be $1 \mathrm{~cm}$, and the smallest grain size $a_{\min }$ is chosen to be 0.1 , 1 or $10 \mu \mathrm{m}$. The $0.1 \mu \mathrm{m}$ size is typical from dust coagulation models (e.g., Birnstiel et al. 2011), and larger sizes are chosen to mimic the effect of grain growth. ${ }^{5}$ The total dust mass ratio is set to $10^{-2}$, and calculations are conducted for individual dust size bins.

In our calculations of volatile evolution, the dust density profile is fixed based on the steady state profile (4). Although the steady-state assumption may not perfectly hold, we note that the only essential requirement of the mechanism we study is that the bulk of the dust settles to the cold midplane layer within the atmospheric snow line (in our model within $\pm 2 \mathrm{H}$ about the midplane). Since the stopping time of grains increases toward disk surface, the timescale for dust to settle to within $2 \mathrm{H}$ is much shorter. Although they do not necessarily achieve a fully steady state distribution, we find that for grain size $\gtrsim 0.1 \mu \mathrm{m}$, the settling timescale above $z \sim 2 H$ is within $1 \mathrm{Myr}$, which suffices for our purpose.

\subsection{Volatile Adsorption and Desorption on Grains}

Volatiles interact with dust grains via adsorption/desorption. The adsorption rate of gas phase CO onto grains of size $a$ is given by

$$
R_{a d}(a) \approx v_{\mathrm{th}} \pi a^{2} n_{d}(a) n_{\mathrm{co}[\mathrm{g}]},
$$

where $v_{\text {th }}$ is the thermal velocity of $\mathrm{CO}, n_{\mathrm{co}[\mathrm{g}]}$ is the gas phase $\mathrm{CO}$ number density. The sticking coefficient is approximately 1 and is left out (Bisschop et al. 2006). Total adsorption rate is a summation over the grain size distribution.

For this work, it suffices to only consider thermal desorption, whose rate is given by (Hasegawa et al. 1992)

$$
R_{\mathrm{des}}(a)=n_{\mathrm{co}[\mathrm{m}]}(a) \nu_{0} \exp \left[-E_{D} / k_{\mathrm{B}} T\right] / \operatorname{Max}\left(1, N_{\text {layer }}\right)
$$

where $n_{\mathrm{co}[\mathrm{m}]}(a) d a$ is number density of adsorbed $\mathrm{CO}$ molecules (mantles) on the surface of dust grains between size $a$ and $a+d a . \nu_{0}=\left(2 n_{s} E_{D} / \pi^{2} m_{\mathrm{co}}\right)^{1 / 2}$ is the characteristic vibration frequency with surface density of adsorption sites $n_{s} \simeq 1.5 \times 10^{15} \mathrm{~cm}^{-2}, m_{\text {co }}$ is $\mathrm{CO}$ mass and we take the $\mathrm{CO}$ binding energy $E_{D}=1150 \mathrm{~K}$. While the exact value of binding energy depends on grain surface properties and can be as low as $\sim 850 \mathrm{~K}$ (Öberg et al. 2005), it does not affect the physics we present here. The extra factor $N_{\text {layer }}=n_{\mathrm{co}[\mathrm{m}]}(a) / 4 \pi a^{2} n_{s} n_{d}(a)$ is the ice layer on dust grains. For ices thicker than monolayer, only the top molecular layer is available for desorption. Note that we assume that volatile freeze-out does not appreciably change the dust grain sizes.

\subsection{D Evolution Model}

We solve time-dependent $1 \mathrm{D}$ evolution equations for gasphase $\mathrm{CO}$ and the mantle-phase $\mathrm{CO}$ for individual dust size bins. The equation for the gas-phase $\mathrm{CO}$ number density reads

$$
\frac{\partial n_{\mathrm{co}[\mathrm{g}]}}{\partial t}=-\nabla \cdot j_{\mathrm{co}[\mathrm{g}]}+\sum_{a}\left[R_{\mathrm{des}}(a)-R_{a d}(a)\right] d a,
$$

\footnotetext{
5 Although the dust coagulation model of Birnstiel et al. (2011) gives a set of broken power laws instead of a single power law, we will show in Section 3.3 that the details of the size distribution do not affect our main results.
}

where the $\mathrm{CO}$ diffusive flux $j_{\mathrm{co}[\mathrm{g}]}$ is given by

$$
j_{\mathrm{co}[\mathrm{g}]}=-n_{\mathrm{g}} D_{z} \frac{\partial}{\partial z}\left(\frac{n_{\mathrm{co}[\mathrm{g}]}}{n_{\mathrm{g}}}\right) \text {, }
$$

with $n_{\mathrm{g}}$ being gas number density.

The evolution for mantle-phase $\mathrm{CO}$ associated with grain size $a$ is given by

$$
\begin{aligned}
\frac{\partial n_{\mathrm{co}[\mathrm{m}]}(a)}{\partial t}= & -\nabla \cdot\left[n_{\mathrm{co}[\mathrm{m}]}(a) v_{d}+j_{\mathrm{co}[\mathrm{m}]}(a)\right] \\
& +R_{a d}(a)-R_{\mathrm{des}}(a),
\end{aligned}
$$

where the diffusive flux follows from dust dynamics

$$
j_{\mathrm{co}[\mathrm{m}]}(a)=-\frac{n_{\mathrm{g}} D_{z}}{S_{\mathrm{c}}} \frac{\partial}{\partial z}\left(\frac{n_{\mathrm{co}[\mathrm{m}]}(a)}{n_{\mathrm{g}}}\right) .
$$

In this formulation, we assume the mantle $\mathrm{CO}$ abundance at each height for each grain size is single-valued, representing the mean value. In reality, mantle-phase $\mathrm{CO}$ abundance on each grain can be different depending on individual grain trajectories. However, such further complication can be essentially absorbed into the desorption prescription via Equation (6), and should not affect the physics we address in this paper.

At $t=0$, we assume $\mathrm{CO}$ molecules are all in the gas phase and are well mixed with the bulk gas. Prescribing fixed turbulence and dust background profile as discussed earlier, the equations are evolved for $1 \mathrm{Myr}$.

\section{Results}

We discuss the results of our calculations in this section. We start from the simplest example with a single grain size, and then consider the more realistic case with a grain size distribution.

\subsection{Time Evolution}

In Figure 3, we show the time evolution of gas/mantle-phase $\mathrm{CO}$ number densities for fixed grain size $a=1 \mu \mathrm{m}$ at $50 \mathrm{au}$ using layered $\alpha_{z}$ profile. At the start of the model, $\mathrm{CO}$ freezes out rapidly in the midplane, while it remains almost completely in gas phase in the warmer surface layer, with the atmospheric snow line located at $z \sim 2 H$. This sharpness of the transition is the result of extremely sensitive temperature dependence of the freeze-out process.

Most existing chemistry models ignore turbulent diffusion, which is equivalent to removing the divergence terms in Equations (7) and (9). In this case, the results correspond to the right panel of Figure 3, where the gas phase $\mathrm{CO}$ abundance is entirely determined by the adsorption/desorption process of the initial CO reservoir, and shows very little time evolution.

Including turbulent diffusion (left panel of Figure 3), we see that $\mathrm{CO}$ at the surface layer is gradually depleted over the timescale of $10^{5-6}$ years, accompanied by the enhancement of mantle phase $\mathrm{CO}$ in the midplane. This confirms the picture we outlined at the beginning of Section 2. By $1 \mathrm{Myr}$, we see that the gas-phase $\mathrm{CO}$ in the surface layer is depleted by a factor of more than 10, which is comparable to the observed level of $\mathrm{CO}$ depletion in PPDs.

Finally, we note that in this calculation the gas and mantle phase $\mathrm{CO}$ profiles have reached an approximate steady-state right around $1 \mathrm{Myr}$, where the downward flux of gas-phase $\mathrm{CO}$ 

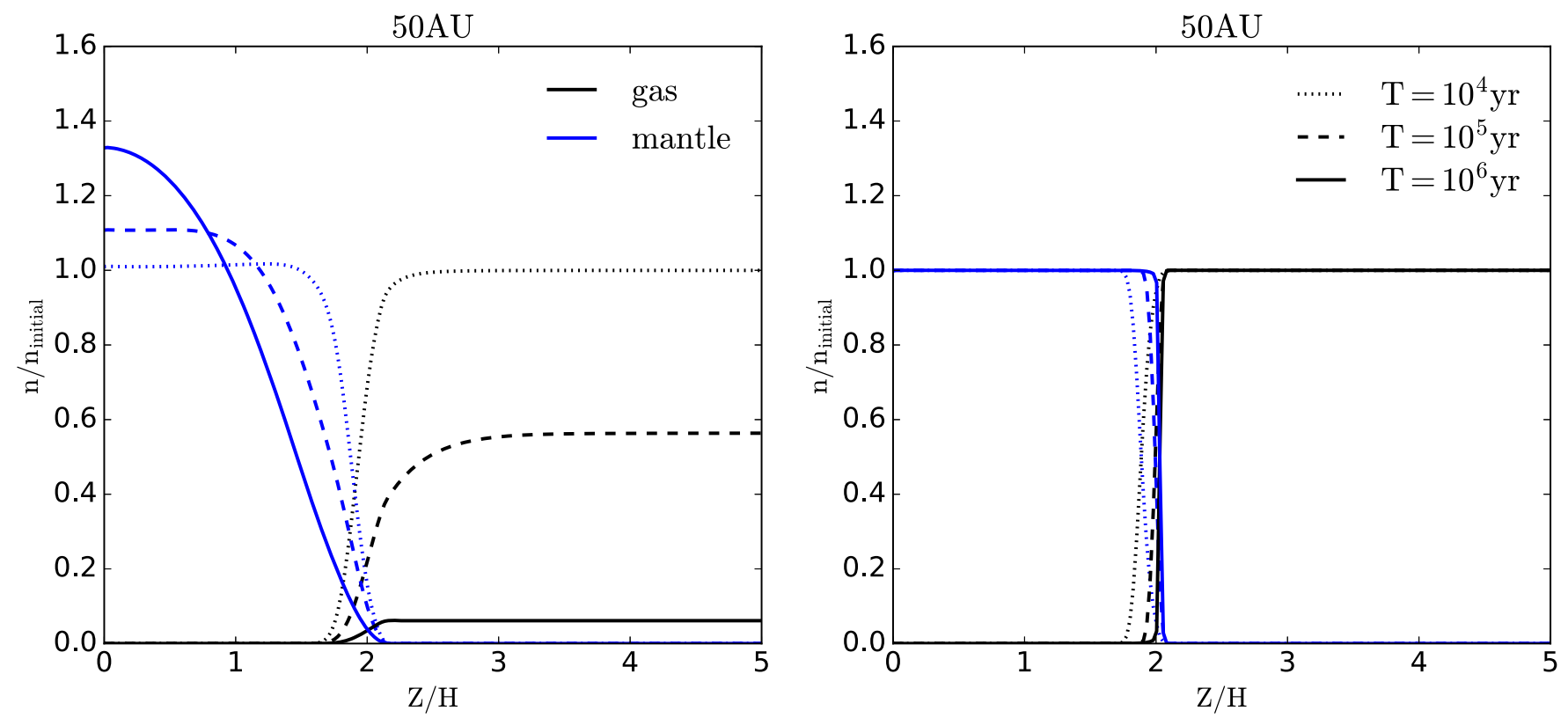

Figure 3. Time evolution of gas phase (black lines) and mantle phase (blue lines) CO number densities (normalized by initial gas phase CO number density) assuming single sized $1 \mu \mathrm{m}$ dust in the layered $\alpha_{z}$ profile at $50 \mathrm{au}$. Left/right panel corresponds to calculations that include/exclude turbulent diffusion of CO. Different line styles correspond to $10^{4}$ years (dotted lines), $10^{5}$ years (dashed lines), and $10^{6}$ years (solid lines).

balances the upward flux of mantle-phase CO. The level of gasphase $\mathrm{CO}$ depletion in steady state is mainly determined by the dust density gradient across the atmospheric snow line, reflecting the level of dust settling. Typically, this steady state is achieved over a few times the turbulent diffusion timescale at the atmospheric snow line.

\subsection{Conditions for Strong CO Depletion}

The example in the previous subsection is to a certain extent optimized. We now discuss under what conditions $\mathrm{CO}$ depletion is efficient by conducting calculations with different grain sizes, disk radius and turbulence profiles. The results after evolving for $1 \mathrm{Myr}$ are shown in Figure 4.

We find that the most stringent requirement to achieve strong $\mathrm{CO}$ depletion is a sufficiently weak turbulence in the midplane so that the vast majority of dust grains can settle within the atmospheric snow line. This condition is demonstrated from two aspects in Figure 4. First, all calculations with strong turbulence (constant $\alpha_{z}=10^{-2}$ ) show essentially no CO depletion. This is because strong turbulence stirs up dust grains to the warmer disk surface, where $\mathrm{CO}$ mantles desorb and return to the gas phase. In steady state, there is an efficient $\mathrm{CO}$ circulation with downward turbulent mixing in the gas phase and upward turbulent diffusion of icy grains. The cancellation of the two effects lead to an outcome that is similar to the case without turbulent mixing. On the other hand, with weak midplane turbulence $\left(\alpha_{z}=10^{-4}\right.$ or the layered profile case), all calculations show a certain level of $\mathrm{CO}$ depletion in the disk surface. Second, more loosely coupled grains lead to more significant depletion, and vice versa. Our calculation with $a=0.1 \mu \mathrm{m}$ shows only a low level of $\mathrm{CO}$ depletion, because these grains only partially settle to the cold midplane region, and instead regularly return to the warmer disk surface.

Too much settling slows down $\mathrm{CO}$ depletion, however. If dust grains concentrate too close to the disk midplane, the $\mathrm{CO}$ depletion rate is reduced because of decreased surface area and therefore longer freeze-out timescales at the $\mathrm{CO}$ atmospheric snowline. Upon reaching steady state, depletion of gas-phase $\mathrm{CO}$ would be more complete, but this may never be achieved, depending on the disk lifetime.

The competing effects of settling on $\mathrm{CO}$ depletion after $1 \mathrm{Myr}$ are readily seen when comparing models run at 50 and $100 \mathrm{au}$. Compared to $50 \mathrm{au}$, grains at $100 \mathrm{au}$ of a fixed size are more loosely coupled (having larger $\tau_{s}$ ). We see that for $0.1 \mu \mathrm{m}$ grains, more $\mathrm{CO}$ depletion is achieved at $100 \mathrm{au}$ compared to $50 \mathrm{au}$, which is because they settle more. For $1 \mu \mathrm{m}$ grains, the depletion is slightly less at $100 \mathrm{au}$ after $1 \mathrm{Myr}$ because the larger grains largely settle far below the atmospheric snowline, leading to a longer $\mathrm{CO}$ depletion timescale, as discussed above. If we continue to evolve the system for $\sim 1-2 \mathrm{Myr}$, more $\mathrm{CO}$ depletion can be achieved than in the 50 au case.

Strong turbulence at the disk surface enhances gas-phase $\mathrm{CO}$ depletion. The turbulent mixing timescale is given by $t_{\text {mix }} \simeq H^{2} / D_{z} \sim\left(\alpha_{z} \Omega_{K}\right)^{-1}$. Freeze-out of CO brought from disk surface primarily occurs around the atmospheric snow line, in our case at slightly above $z=2 \mathrm{H}$. For constant $\alpha_{z}=10^{-4}$, the mixing timescale is the same at all heights. As a result, depletion of $\mathrm{CO}$ occurs first at the atmospheric snow line, and then propagates toward disk upper layer, leading to a slow depletion toward disk surface. With a layered $\alpha_{z}$ profile, much more efficient turbulent mixing at the disk surface homogenize the gas-phase $\mathrm{CO}$ distribution there. As a result, $\mathrm{CO}$ depletion proceeds almost simultaneously through the entire disk surface column.

\subsection{Importance of Dust Distribution Characteristics}

In this subsection, we consider the effects of dust size distributions with the three size ranges mentioned in Section 2.1. They differ in the smallest grain size $a_{\min }$. Theoretically, we expect the smallest grains to play the dominant role in the outcome of $\mathrm{CO}$ depletion. This is because the smallest grains generally dominate the total dust surface area, and are more suspended and therefore directly accessible to the fresh $\mathrm{CO}$ molecules brought down from the disk surface. 

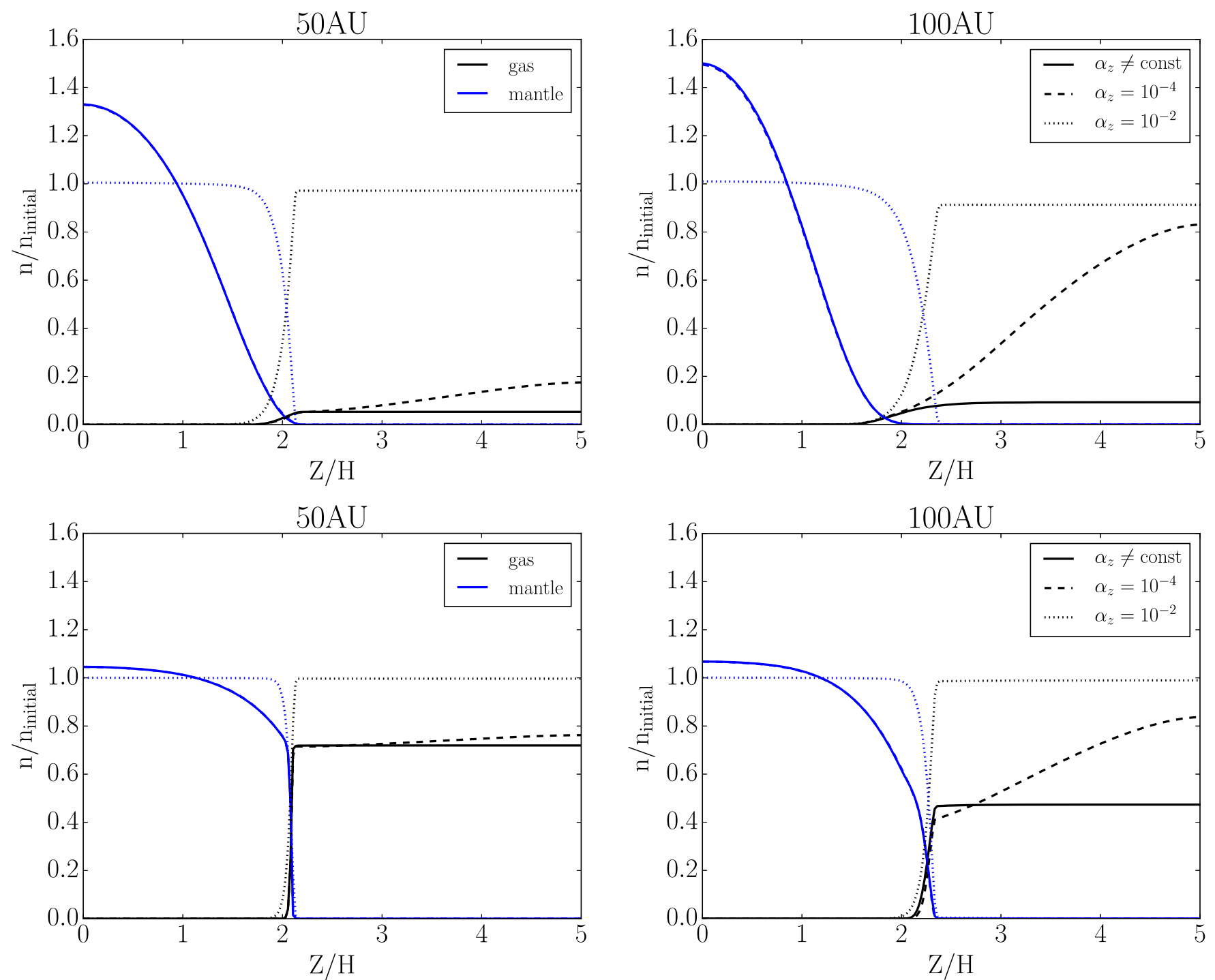

Figure 4. Vertical profiles of gas phase (black) and mantle phase (blue) CO number densities after 1 Myr evolution (normalized to initial CO number density) at 50 au (left) and 100 au (right) with layered $\alpha_{z}$ profile (solid), constant $\alpha_{z}=10^{-4}$ (dashed line), and constant $\alpha_{z}=10^{-2}$ (dotted line). Grain size is fixed at $0.1 \mu \mathrm{m}$ on the top panels and $1 \mu \mathrm{m}$ on the bottom panels.

Larger grains contribute less or even negligibly to $\mathrm{CO}$ freezeout because they have much smaller total surface area. Also, they often settle well below the atmospheric snow line and therefore have little direct access to the gas-phase CO.

Figure 5(a) shows gas-phase and total mantle-phase $\mathrm{CO}$ number densities after $1 \mathrm{Myr}$ evolution with the three different size distributions. We can see in cases with $a_{\min }=0.1 \mu \mathrm{m}$ (solid line) and $1 \mu \mathrm{m}$ (dashed line), the overall level of $\mathrm{CO}$ depletion is comparable with their single grain size $(0.1 \mu \mathrm{m}$ and $1 \mu \mathrm{m})$ counterparts shown in Figure 4, which confirms our expectations. Increasing $a_{\mathrm{min}}$ further to $10 \mu \mathrm{m}$ leads to slower depletion (dotted line). In this case, even the smallest grains are large enough to settle well below the atmospheric snow line (see Section 3.2), leading to a longer depletion timescale.

Figures 5(b)-(d) show the distribution of mantle-phase CO as a function of grain size and vertical height. We see that most of the mantle-phase $\mathrm{CO}$ is in the smallest grains, which confirms our previous discussion that $\mathrm{CO}$ depletion is dominated by the smallest grains. We note that $a \sim 1 \mu \mathrm{m}$ is a critical grain size in our calculations. For $a \lesssim 1 \mu \mathrm{m}$, a considerable fraction of grains stay outside the atmospheric snowline. In the case of $a_{\min }=10 \mu \mathrm{m}$, most mantle-phase CO is well within the atmospheric snow line, which simply reflects the deficit of grains at the atmospheric snow line. We see that the $a_{\min }=1 \mu \mathrm{m}$ case leads to fastest CO depletion. It suggests that $\mathrm{CO}$ depletion is the most efficient when the population of grains containing most of the surface area (smallest grains) settle just within the atmospheric snow line.

Note that we have assumed a fixed grain size in our calculations. In reality, coagulation of small grains into larger grains, followed by the settling of larger grains, may increase the mantle-phase $\mathrm{CO}$ abundances on larger grains than shown in Figure 5.

Overall, with a grain size distribution, the rate of $\mathrm{CO}$ depletion is comparable with the case of a single-sized grain of smallest size. Over a $1 \mathrm{Myr}$ timescale, depletion of $\mathrm{CO}$ by a factor of $\sim 2$ can be achieved for the more conservative case with the smallest grain size being $0.1 \mu \mathrm{m}$, and more complete depletion can be achieved if grains grow bigger. We thus conclude that, as long as the conditions discussed in the previous subsection are satisfied, turbulent diffusion is a robust mechanism for volatile depletion. 

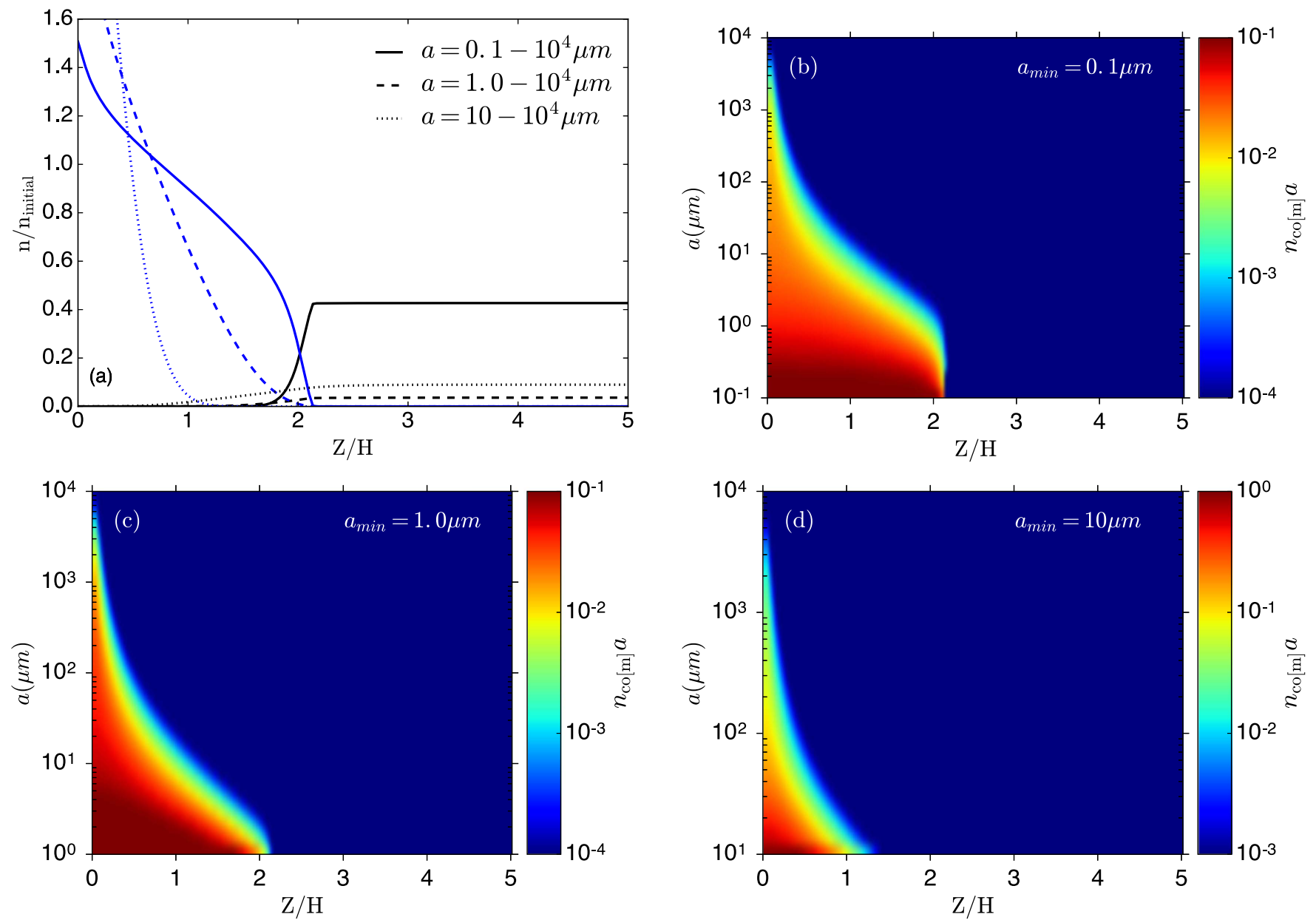

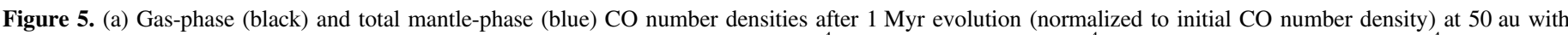

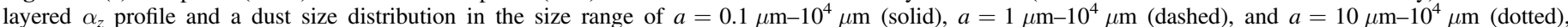

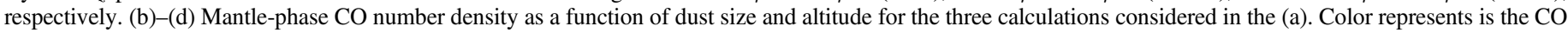

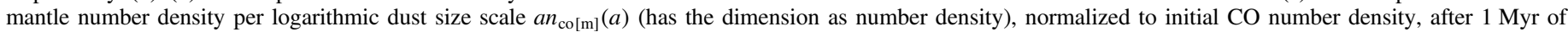
evolution. (b)-(d) correspond to calculations with smallest grain size of $a_{\min }=0.1 \mu \mathrm{m}, 1 \mu \mathrm{m}$, and $10 \mu \mathrm{m}$, respectively.

\section{Summary and Discussion}

In this paper, we have presented a simple semi-analytical model which demonstrates that, as a result of dust settling and turbulent diffusion, $\mathrm{CO}$ in the warm surface layer of the outer regions of PPDs is subject to turbulent diffusion into the cold midplane and subsequent depletion.

The most important condition for turbulent-diffusion mediated $\mathrm{CO}$ depletion is that midplane turbulence must be sufficiently weak that the bulk of the small grains that dominate the surface area can settle within the atmospheric snow line. The process is facilitated by stronger turbulence in the disk surface layer. Both conditions are likely realizable in the outer regions of PPDs (Perez-Becker \& Chiang 2011; Simon et al. 2013; Bai 2015).

Our results suggest that turbulent diffusion likely contributes to the observed carbon (especially $\mathrm{CO}$ ) depletion in PPDs, particularly in the disk surface region (e.g., Du et al. 2015). Its contribution depends on the level of midplane turbulence and grain size distribution, and can be a factor of a few to more than one order of magnitude. In reality, we expect that additional mechanisms also contribute to carbon depletion. Conversion of carbon to complex organic molecules likely yields a factor of a few of depletion over disk lifetime (Bergin et al. 2014; Yu et al. 2016). Gas removal from disk wind likely contributes another factor of two to a few to the reduced gas-to-dust mass ratio given that wind mass loss rate is comparable to the mass accretion rate (Bai 2016). Altogether, these processes are likely to be able to account for a wide range (two orders of magnitude) of the $\mathrm{CO}$ depletion factor, and/or the apparent gas-to-dust ratio inferred from observations.

While the mechanism of $\mathrm{CO}$ depletion in the outer disk discussed here is similar to previous studies on the depletion of water vapor in the inner disk (Meijerink et al. 2009; Ros \& Johansen 2013; Krijt et al. 2016), there are important differences. One may not directly apply our calculation results to the inner disk, and vice versa.

We first note that the level of turbulence in the inner region of PPDs is likely very weak, yet highly uncertain. On the one hand, the MRI is largely suppressed (e.g., Bai \& Stone 2013), yet some turbulence is needed to keep small grains suspended to match the observed disk spectral energy distributions (SEDs), which may be attributed to certain poorly understood hydrodynamic instabilities (e.g., Turner et al. 2014, p. 411). We choose to focus on the outer regions of PPDs, where layered turbulence structure is likely a natural outcome of the MRI operating in the surface FUV layer (Perez-Becker \& Chiang 2011). 
Another important difference between the inner and outer regions of PPDs is the timescale. The inner disk is characterized by fast collision and grain growth timescales, and sub-micron grains are mainly the product of destructive collisions of large grains. Recently, Krijt \& Ciesla (2016) showed that because of their short collisional coagulation timescale, small grains can be effectively trapped in the midplane region without diffusing to disk upper layers. This effect can substantially enhance volatile depletion, and in the case of water, such depletion further enhances grain growth (Ros \& Johansen 2013; Krijt et al. 2016).

We have ignored the growth and collisional evolution of grains in our calculations. While this is less self-consistent, we note that in the outer disk, grain growth is much slower and is found to be drift-limited rather than fragmentation limited (e.g., Birnstiel et al. 2012). Therefore, the smallest grain population in the outer disk is mostly primordial rather than from collisional fragmentation. As we have mentioned in Section 2.1, it takes $\sim 1 \mathrm{Myr}$ for $\sim 0.1 \mu \mathrm{m}$ grains to settle to within $\sim 2 \mathrm{H}$ at $\sim 50 \mathrm{au}$. We also estimate a similar $\sim \mathrm{Myr}$ timescale for collisions between sub-micron grains at that height (which is more relevant for $\mathrm{CO}$ depletion, instead of midplane). Therefore, because of the long timescale in the outer disk, we expect grain growth to only play a minor role in the outer disk physics discussed in this work.

As an initial effort, we focus on the physics of the mechanism using a simple 1D model, which captures the essence of the problem. One important limitation is that we have ignored the radial dimension, where grains undergo radial drift, and the disk itself evolves over Myr timescales (Takeuchi $\&$ Lin 2002). We note that significant improvement in our knowledge of disk evolution is needed before a more reliable dust transport model can be made, especially given the prevalence of disk substructures that has been realized in the recent years (e.g., ALMA Partnership et al. 2015; Andrews et al. 2016; Nomura et al. 2016; Zhang et al. 2016). Regardless of the details of radial dust transport, we expect our main conclusions to be robust as long as the vertical profile of turbulence does not vary significantly with radius in the outer disk.

Overall, our work has demonstrated the importance of incorporating more realistic disk dynamics (i.e., turbulent diffusion) into models of volatile evolution (e.g., Ciesla \& Cuzzi 2006). The outcome is important for determining, for instance, the location of the volatile condensation fronts/snow lines (Öberg et al. 2011; Qi et al. 2013; Piso et al. 2015), and volatile delivery to planets which would affect the planets' bulk and atmospheric composition (Madhusudhan et al. 2011). More generally, volatiles play an important role in the overall disk chemistry (Henning \& Semenov 2013). As initially pursued in Semenov \& Wiebe (2011), we expect future studies of PPD chemical evolution to pay more attention to, and eventually benefit from, incorporating more realistic PPD gas dynamics.

We thank Chunhua Qi, Fred Ciesla, Til Birnstiel and Ted Bergin for helpful discussions, and Mihkel Kama, Klaus Pontoppidan, Eugene Chiang and an anonymous referee for useful comments that greatly improve the paper. X.N.B. acknowledges support from Institute for Theory and Computation, Harvard-Smithsonian Center for Astrophysics. K.I.Ö. acknowledges funding through a Packard Fellowship for Science and Engineering from the David and Lucile Packard Foundation.

\section{References}

ALMA Partnership, Brogan, C. L., Pérez, L. M., et al. 2015, ApJL, 808, L3 Andrews, S. M. 2015, PASP, 127, 961

Andrews, S. M., \& Williams, J. P. 2007, ApJ, 659, 705

Andrews, S. M., Wilner, D. J., Hughes, A. M., Qi, C., \& Dullemond, C. P. 2009, ApJ, 700, 1502

Andrews, S. M., Wilner, D. J., Zhu, Z., et al. 2016, ApJL, 820, L40

Ansdell, M., Williams, J. P., van der Marel, N., et al. 2016, arXiv:1604.05719 Bai, X.-N. 2015, ApJ, 798, 84

Bai, X.-N. 2016, ApJ, 821, 80

Bai, X.-N., \& Stone, J. M. 2011, ApJ, 736, 144

Bai, X.-N., \& Stone, J. M. 2013, ApJ, 769, 76

Balbus, S. A., \& Hawley, J. F. 1991, ApJ, 376, 214

Bergin, E. A., Cleeves, L. I., Crockett, N., \& Blake, G. A. 2014, FaDi, 168 arXiv:1405.7394

Bergin, E. A., Du, F., Cleeves, L. I., et al. 2016, ApJ, 831, 101

Bergin, E. A., Cleeves, L. I., Gorti, U., et al. 2013, Natur, 493, 644

Birnstiel, T., Klahr, H., \& Ercolano, B. 2012, A\&A, 539, A148

Birnstiel, T., Ormel, C. W., \& Dullemond, C. P. 2011, A\&A, 525, A11

Bisschop, S. E., Fraser, H. J., Öberg, K. I., van Dishoeck, E. F., \& Schlemmer, S. 2006, A\&A, 449, 1297

Bohlin, R. C., Savage, B. D., \& Drake, J. F. 1978, ApJ, 224, 132

Bruderer, S., van Dishoeck, E. F., Doty, S. D., \& Herczeg, G. J. 2012, A\&A, 541, A91

Carr, J. S., \& Najita, J. R. 2008, Sci, 319, 1504

Ciesla, F. J., \& Cuzzi, J. N. 2006, Icar, 181, 178

Du, F., Bergin, E. A., \& Hogerheijde, M. R. 2015, ApJL, 807, L32

Eisner, J. A., Bally, J. M., Ginsburg, A., \& Sheehan, P. D. 2016, ApJ, 826, 16 Epstein, P. S. 1924, PhRv, 23, 710

Favre, C., Cleeves, L. I., Bergin, E. A., Qi, C., \& Blake, G. A. 2013, ApJL, 776, L38 Frerking, M. A., Langer, W. D., \& Wilson, R. W. 1982, ApJ, 262, 590

Glassgold, A. E., Meijerink, R., \& Najita, J. R. 2009, ApJ, 701, 142

Glassgold, A. E., Najita, J., \& Igea, J. 2004, ApJ, 615, 972

Gorti, U., Hollenbach, D., \& Dullemond, C. P. 2015, ApJ, 804, 29

Hasegawa, T. I., Herbst, E., \& Leung, C. M. 1992, ApJS, 82, 167

Henning, T., \& Semenov, D. 2013, ChRv, 113, 9016

Kama, M., Bruderer, S., Carney, M., et al. 2016a, A\&A, 588, A108

Kama, M., Bruderer, S., van Dishoeck, E. F., et al. 2016b, A\&A, 592, A83

Kamp, I., \& Dullemond, C. P. 2004, ApJ, 615, 991

Krijt, S., \& Ciesla, F. J. 2016, ApJ, 822, 111

Krijt, S., Ciesla, F. J., \& Bergin, E. A. 2016, arXiv:1610.06463

Madhusudhan, N., Harrington, J., Stevenson, K. B., et al. 2011, Natur, 469, 64

Mathis, J. S., Rumpl, W., \& Nordsieck, K. H. 1977, ApJ, 217, 425

Meijerink, R., Pontoppidan, K. M., Blake, G. A., Poelman, D. R., \& Dullemond, C. P. 2009, ApJ, 704, 1471

Miotello, A., Bruderer, S., \& van Dishoeck, E. F. 2014, A\&A, 572, A96

Nomura, H., Tsukagoshi, T., Kawabe, R., et al. 2016, ApJL, 819, L7

Öberg, K. I., Murray-Clay, R., \& Bergin, E. A. 2011, ApJL, 743, L16

Öberg, K. I., van Broekhuizen, F., Fraser, H. J., et al. 2005, ApJL, 621, L33

Perez-Becker, D., \& Chiang, E. 2011, ApJ, 727, 2

Piso, A.-M. A., Öberg, K. I., Birnstiel, T., \& Murray-Clay, R. A. 2015, ApJ, 815,109

Qi, C., Öberg, K. I., Wilner, D. J., et al. 2013, Sci, 341, 630

Ripple, F., Heyer, M. H., Gutermuth, R., Snell, R. L., \& Brunt, C. M. 2013, MNRAS, 431, 1296

Ros, K., \& Johansen, A. 2013, A\&A, 552, A137

Rosenfeld, K. A., Andrews, S. M., Hughes, A. M., Wilner, D. J., \& Qi, C. 2013, ApJ, 774, 16

Salyk, C., Pontoppidan, K. M., Blake, G. A., et al. 2008, ApJL, 676, L49

Schwarz, K. R., Bergin, E. A., Cleeves, L. I., et al. 2016, ApJ, 823, 91

Semenov, D., \& Wiebe, D. 2011, ApJS, 196, 25

Shakura, N. I., \& Sunyaev, R. A. 1973, A\&A, 24, 337

Simon, J. B., Bai, X.-N., Armitage, P. J., Stone, J. M., \& Beckwith, K. 2013, ApJ, 775, 73

Stevenson, D. J., \& Lunine, J. I. 1988, Icar, 75, 146

Takeuchi, T., \& Lin, D. N. C. 2002, ApJ, 581, 1344

Turner, N. J., Fromang, S., Gammie, C., et al. 2014, in Protostars and Planets

VI, ed. H. Beuther et al. (Tucson, AZ: Univ. Arizona Press), 411

Walsh, C., Millar, T. J., \& Nomura, H. 2010, ApJ, 722, 1607

Williams, J. P., \& Best, W. M. J. 2014, ApJ, 788, 59

Williams, J. P., \& Cieza, L. A. 2011, ARA\&A, 49, 67

Youdin, A. N., \& Lithwick, Y. 2007, Icar, 192, 588

Yu, M., Willacy, K., Dodson-Robinson, S. E., Turner, N. J., \& Evans, N. J., II 2016, ApJ, 822, 53

Zhang, K., Bergin, E. A., Blake, G. A., et al. 2016, ApJL, 818, L16 